\title{
Inaction on climate change projected to reduce European life expectancy
}

\author{
Mathew E. Hauer \\ Department of Sociology, Florida State University \\ and \\ Alexis R. Santos \\ Human Development and Family Studies, Pennsylvania State University
}

April 13, 2019

\begin{abstract}
Climate change-related excess mortality estimates clearly demonstrate a dramatic impact on public health and human mortality. However, life expectancy at birth is more easily communicated and understood by the public. By properly situating climate change mortality within the contexts of life expectancy, we better represent the cost of climate change on longevity. In this paper, we convert excess mortality estimates due to increases in extreme weather from climate change into potential reductions in life expectancy at birth in thirty-one European countries. We project climate change extremes to reduce life expectancy at birth by 0.24 years for the average European country with differences in excess of 1.0 years in some countries by the end of the century. We only estimate the impact of mortality directly related to climate extremes, making our estimates conservative. Thus, the cost of inaction on climate change could approach, and likely to exceed, one year of life in some European countries.
\end{abstract}

Keywords: climate change, life expectancy, mortality, Europe 


\section{Main Text}

Climate change's implications on humanity go far beyond estimates of economic damages (Hsiang et al. 2017), estimates of displacement (Rigaud et al. 2018), or human conflict (Barnett \& Adger 2007) but have the potential to contribute to the loss of human life (Forzieri et al. 2017, Pachauri et al. 2014). As impact quantification studies move further into the social sciences, properly quantifying and conveying the impact of climate change on public health is of increasing importance (Melillo et al. 2014, Cloyd et al. 2016).

Scholars have long estimated the mortality risks associated with climate change and typically use excess or extra mortality (Forzieri et al. 2017, Wilson et al. 2017, McMichael et al. 2006, Zanobetti et al. 2012). For example, Forzieri et al. (2017) project climate change extremes to increase European mortality by $150,000+$ deaths per year by the end of the century. Although such estimates are useful, the excess mortality estimates are rather sterile - one death is a tragedy, a million deaths a statistic - and difficult to relate to on a personal level. Life expectancy at birth $\left(e_{0}\right)$, on the other hand, provides potent comparisons of mortality vectors and converts excess mortality into an intuitively understood metric, relatable to everyone. To our knowledge, no studies exist that comprehensively examine the potential reductions of life expectancy due to climate change. Without properly situating the potential loss of life within the contexts of metrics such as life expectancy, we risk underestimating and misreporting the impact of climate change on human mortality.

Using published data on excess mortality (Forzieri et al. 2017), we connect climate change excess mortality to life expectancy in a mortality model. By estimating the increase in age-specific mortality rates associated with previously published excess death estimates, we can assess how much climate change could reduce the anticipated longevity of the average person in thirty-one European countries. This approach allows us to quantify the impact of climate change on human longevity and answer the following question: What is the cost of inaction on climate change on human longevity? Our results situate climate change mortality within the broader context of human mortality and can be used to inform public health interventions and adaptation measures to prevent such futures.

Our results suggest that climate change could emerge as a potent public health threat in the 21st century. Mitigation strategies (ie reductions in greenhouse gas emissions) and 
adaptation strategies (ie outreach efforts, retrofitting public buildings, etc.) would help prevent this new public health concern.

\section{Methods and Materials}

\subsection{Data}

We estimate changes in life expectancy by combining three primary data sets. First, we use the projected population's by age in conjunction with projected age-specific mortality rates from the United Nations' World Population Prospects (United Nations 2015). This data provides both the future populations exposed to future climate hazards and the data necessary to calculate the difference in life expectancy between baseline, projected mortality and mortality due to climate change. Second, we use projections of excess mortality due to meteorological hazards related to climate change from Forzieri et al. (2017). This data provides the number of projected deaths to climate change by the end of the century. And third, we use cause-of-death distributions from the Global Burden of Disease study (GBD) (of Disease Study 2015 2017, Wang et al. 2012) to distribute Forzieri et al's (2017) projected death totals.

Forzieri et al. (2017) combine data from the International Disaster Database (EMCAT) and the Natural Catastrophe Statistics tool (NatCatSERVICE) to create exposure and fatality statistics related to six weather-related hazards - heatwaves, cold waves, droughts, wildfires, river and coastal floods, and windstorms - and estimate excess mortality associated with those hazards during a baseline period of 1981-2010 in 28 European countries. They only considered fatalities directly attributable to the event and exclude increased deaths from common causes that were observed to rise during the event, such as cardiovascular or respiratory deaths $\}^{1}$. While the disaster data are not standardized across country, Forzieri et al. imputed data in incomplete time periods and countries. This imputation

\footnotetext{
1 "The fatality data from the two databases considered is likely to not include increased deaths from common causes that were observed to rise at the population level but for which individual deaths could not be attributed to the event. For example,... increased risk of cardiovascular and respiratory deaths ... may be severely under-reported in the EMDAT and NatCatSERVICE." (see Forzieri et al. 2017, pp. 9, Supplementary Materials).
} 
could mask spatial variability at the sub-national level, but here we use their countryaggregated results. They downscaled these exposures rates to $1 \mathrm{~km}$ grid cells and integrated them into small-area demographic projections for Europe out to 2100 that correspond to a middle-of-the-road socioeconomic scenario (SSP2 with medium fertility, medium mortality, medium migration, and the Global Education Trend education scenario (Jiang 2014, Samir \& Lutz 2017, O'Neill et al. 2017)) and based their projected mortality on extrapolations of their calculated exposures. Their results represent business-as-usual climate change and human development without incorporating potential adaptations to climate change. They found a potential $150,000+$ climate change related fatalities per year by the mid 2080s with climate change contributing to $90 \%$ of mortality rise as opposed to population changes. The number of future anticipated deaths are available in their Supplementary Table S8 (Forzieri et al. 2017) and provide the magnitude of deaths for our study.

The Forzieri et al. (2017) data contain only mortality totals and report no age detail, precluding a direct conversion of excess mortality to life expectancy. To convert excess mortality to life expectancy, we allocate excess multi-hazard mortality for each country based on the observed age-specific mortality schedule for environmental heat and cold exposure deaths from the GBD (of Disease Study 2015 2017, Wang et al. 2012). Forzieri et al. found that extreme heat (as opposed to other five weather-related hazards) account for $99 \%$ of the anticipated excess mortality by the end of the century. The GBD is the most comprehensive worldwide data set of epidemiological data and provides cause-specific mortality by age/sex/geography/year for 249 causes of death in 195 countries and territories. We gather data on age-specific mortality rates $\left(m_{x}\right)$ from environmental heat and cold exposure (cause C.2.9) for the period 2006-2015 for each country in the study for age groups $x=0,1,5,10, \ldots 95$. These data provide a mortality schedule to fit the projected climate change excess mortality from Forzieri et al (Forzieri et al. 2017). We assume that the age-specific mortality schedules observed between 2006 and 2015 due to environmental heat and cold exposure in the GBD are likely to remain unchanged in the future. We only use the GBD to distribute the Forzieri et al. deaths by age $\mathrm{q}^{2}$.

With the Forzieri et al data and the GBD we estimate the anticipated age-specific

\footnotetext{
${ }^{2}$ GBD data can be retrieved here: http://ghdx.healthdata.org/gbd-results-tool?params=gbdapi-2017-permalink/b235b96d60b3b5f06f3e080974c415f3
} 
mortality schedules due to climate change in thirty-one European countries but we cannot compare life expectancy differentials in the absence of Climate Change using those two data sets alone. To create baseline mortality schedules and life expectancy, we gather data from the United Nations' World Population Prospects (WPP-2017) (United Nations 2015) for the corresponding European countries in the period 2080-2085 (the mid-point of the period Forzieri et al. report their results (2017)). We use the UN abridged life table data for ages $x=0,1,5,10, \ldots 85$ containing the necessary mortality rates $\left({ }_{n} m_{x}\right)$, the average number of years lived in the age-interval $\left({ }_{n} a_{x}\right)$, and populations $\left({ }_{n} P_{x}\right)$ for populations aged $x$ to $x+n$.

\subsection{Methods}

We derive a number of variables to accomplish our analysis. First, we use the C.2.9 mortality rates $\left({ }_{n} m_{x}\right)$ from the GBD for ages $x=0,1,5,10, \ldots, 95$ with the projected populations aged $x$ to $x+n$ from the UN data in the period 2080-2085 $\left({ }_{n} P_{x}\right)$ in country $i$ to calculate an un-scaled projection of deaths due to C.2.9 $\left({ }_{n} D_{x, i}={ }_{n} m_{x i}^{G B D} \cdot{ }_{n} P_{x}\right)$. We then abridge ${ }_{n} D_{x i}$ to ages $85+$ to match the open-ended age interval found in the UN life tables. With abridged baseline heat/cold deaths, we proportionally rescale ${ }_{n} D_{x, i}$ to sum to the projected excess mortality for each scenario $s(B A S E, L O W, M I D, H I G H)$ for each individual country from Forzieri et al (Forzieri et al. 2017) $\left({ }_{n} \hat{D}_{x, i}\right)$ and calculate new ${ }_{n} m_{x s}^{F O R Z}$. BASE refers to the baseline mortality rates projected by the UN for the period 2080-2085 which do not account for climate change. These calculations combine to create a projected age-specific mortality rates based on Forzieri et. al's excess mortality.

To calculate life expectancy differentials, we add the age-specific Forzieri rates ${ }_{n} m_{x s}^{F O R Z}$ to the projected age-specific mortality rates found in the published UN life tables ${ }_{n} m_{x}$ to create a projected $\hat{m}_{x}=m_{x}+m_{x}^{F O R Z}$. We then calculate life table components ${ }_{n} q_{x},{ }_{n} d_{x}$, $l_{x}, L_{x}, T_{x}$, and ultimately $e_{x}$ identically for each scenario $s$ using standard procedures, substituting ${ }_{n} m_{x}$ with ${ }_{n} \hat{m}_{x}$ where needed:

$$
\begin{gathered}
{ }_{n} q_{x, i, s}=\frac{{ }_{n} m_{x, i, s} \cdot n}{1+\left(n-{ }_{n} a_{x, i}\right) \cdot{ }_{n} m_{x, i, s}} \\
{ }_{n} d_{x, i, s}={ }_{n} l_{x, i, s} \cdot{ }_{n} q_{x, i, s} \\
l_{x, i, s}={ }_{n} l_{x-1, i, s}-{ }_{n} d_{x-1, i, s}
\end{gathered}
$$




$$
\begin{gathered}
L_{x, i, s}=n \cdot{ }_{n} l_{x, i, s}+\left(\left(n-{ }_{n} a_{x, i, s}\right) \cdot{ }_{n} l_{x, i, s}\right) \\
T_{x, i, s}={ }_{n} L_{x, i, s}+{ }_{n} T_{x+1, i, s} \\
e_{x, i, s}={ }_{n} T_{x, i, s} /{ }_{n} l_{x, i, s}
\end{gathered}
$$

We calculate projected life expectancy reductions due to climate change related mortality by subtracting $e_{x, i, s}$ (for scenario $L O W, M I D$, and $H I G H$ ) from $e_{x, i, B A S E}$ as described by (Beltrán-Sánchez et al. 2008).

\subsection{Reproducible Research}

All data and code necessary to reproduce the reported results are licensed under the CCBY-4.0 license and are publicly available in a replication repository located at https: //osf .io/fp52x/?view_only=754d9a72a2ea4f6b8e0c193dc9a590d1.

\section{Results}

We find that climate change could alter life expectancy by -0.24 years $(-2.8$ months $(-0.12$ to -0.39 years $)$ ) in the average European country (Figure 1) by 2100. This reduction is comparable to mortality due to influenza and pneumonia (Arias et al. 2013) in the United States for the year 2000. Although the average European country could see a change of -0.24 years, several countries are likely to experience considerably greater reductions in life expectancy. Spain, for example, could see a reduction of up to 1.44 years $(1.17-1.87)$.

Our results also suggest climate change mortality differentials are likely to unfold along highly uneven geographies (Figure 2). Whereas many Northern European countries could experience negligible impacts on life expectancy, seven European countries could see life expectancy changes in excess of -0.5 years (Spain, Italy, Portugal, France, Slovenia, Croatia, and Luxembourg). This group of countries could see life expectancy changes of more than -1.0 years if climate change hazards are more intense than anticipated. In some countries, climate change could thus become a bigger killer than trachea, bronchus, and lung malignant neoplasms (-0.85 years), acute myocardial infarction (-0.87 years), or all accident related mortality (-0.84 years) (Arias et al. 2013) by the end of the century. 


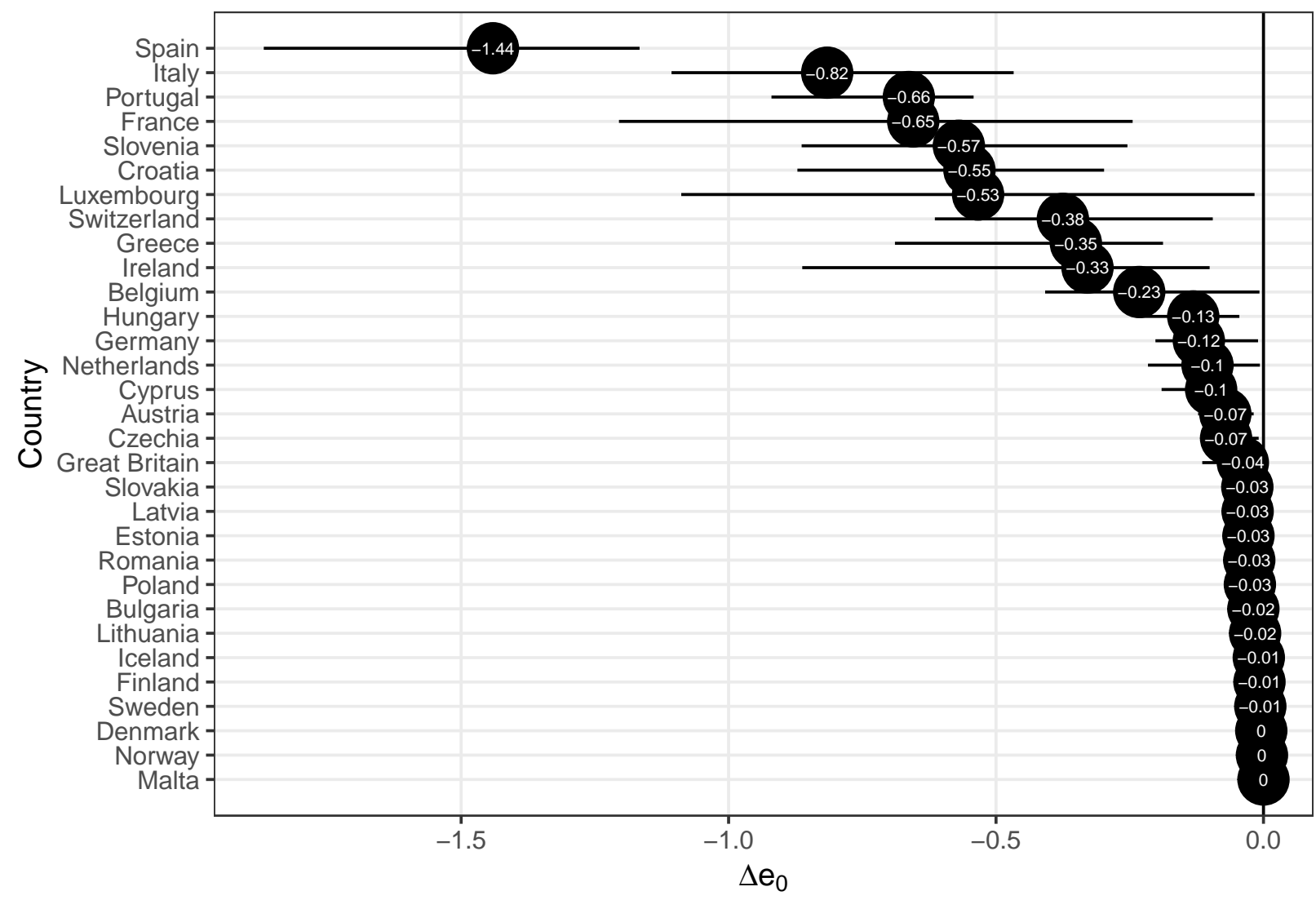

Figure 1: Change in life expectancy at birth $\left(e_{0}\right)$ due to business-as-usual climate change in the 2080s compared to the projected $e_{0}$. We report changes in life expectancies due to climate change for thirty-one European countries. The central values represent the ensemble median while the stems represent the upper and lower bounds of the inter-model climate variability. 


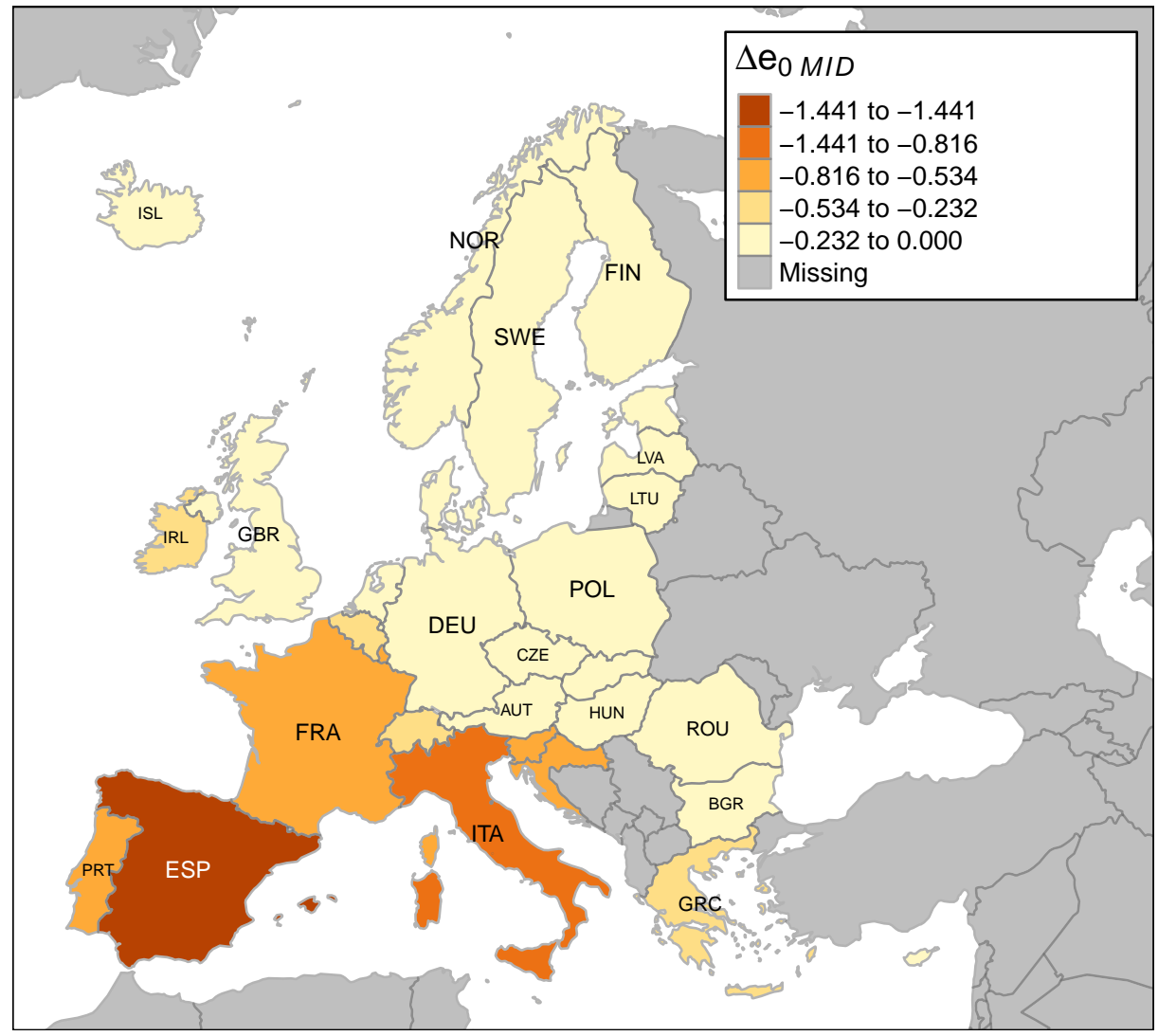

Figure 2: Estimated reduction in life expectancy at birth $\left(e_{0}\right)$ by the 2080s under the $M I D$ scenario. 


\section{Discussion}

In this article, we demonstrate the impact climate change could have on life expectancy at birth due to extreme weather events in thirty-one European countries. Previous studies on climate change and excess mortality potentially miscommunicate the impact climate change could have on human mortality. Contrary to excess mortality estimates, life expectancy is routinely used as a primary metric for communicating overall health outcomes and enjoys widespread use by major international organizations Organization 2015, Marmot et al. 2012, Salomon et al. 2012). Life expectancy and its derivatives are the recommended metrics for population health (Parrish 2010). Additionally, it connects mortality estimates into intuitively understandable metrics, translating global estimates of mortality into personally relatable outcomes. Our work reveals the extent to which climate change could reduce the average person's longevity by the end of the century, expanding our understanding of climate change and public health; thus linking two of the major areas in current developmental and sustainability discussion at both national and international levels (Abel et al. 2016).

Without adaptation measures, our results suggest climate change could emerge as a significant new mortality vector and could pose a major public health threat for some European countries by the end of the century, echoing previous findings (Forzieri et al. 2017, Patz et al. 2005). Life expectancy has steadily risen across the world for the last century (Gerland et al. 2014) and our results suggest that climate change alone could slow these trends in some countries. We expect one European country to see life expectancy reductions of more than one year under the middle scenario (Spain), but if climate change has a greater impact on mortality than anticipated, four European countries (Spain, Italy, France, Luxembourg) could see life expectancy reductions of more than one year with Spain experiencing a reduction of nearly two. These findings highlight the hyperlocalized impacts of climate change (Kendon et al. 2014, Rosenzweig et al. 2010, Forzieri et al. 2017).

Reductions such as these should not be taken lightly. Many of the children born today are likely to still be alive by the end of the century and will be in the age groups (aged $65+$ ) most threatened by the biggest mortality risk associated with climate change Keatinge et al. 2000) - extreme heat. Climate change could thus be one of the most aggressive new 
mortality vectors to emerge over the last quarter-century, representing a major threat to public health in many parts of Europe.

Prospective studies on the emerging threat from climate change rely on linking contemporary mortality with future mortality. However, climate change could reshape future mortality through other causes of death. Climate change affects health behaviors that in turn increase mortality risk through increased alcohol and substance abuse, violent behavior, insecurity, increase in post-traumatic stress due to weather-related trauma, increase in stress due to climate change and schizophrenia, increase in the use of medications that reduce the ability to perspire and sweat, etc. (Patz et al. 2005) The International Classifications of Diseases and Related Health Problems (ICD) does not contain "climate change" as an official cause of death, so we can only speculate that the impact of climate change could be larger than reported here. Although we do not model these potential impacts, our results could thus be considered conservative.

These results should also be considered conservative when compared to the broader impact of climate change on human longevity. The disaster databases that Forzieri et al Forzieri et al. 2017) use in generating their excess mortality estimates probably, though the disaster databases are unclear, only account for the deaths certified as directly caused by these hazards and are unlikely to capture the overall numbers associated with these deaths. If the certification of deaths due to weather extremes is similar to the certification in the present, than our results and those of Forzieri et al reflect the excess mortality directly attributable to climatic extremes. Despite this limitation, the impact of these extremes on $e_{0}$ is considerable, even if conservative.

We also share the concerns of Lee et al. (Lee \& Kim 2017) concerning the business-asusual climatic assumptions. It is likely that many countries and communities will deploy a wide variety of adaptation measures (Haines et al. 2006, Kovats et al. 2003, Ebi et al. 2006). These adaptation measures rely on accurate information about the potential mortality vectors. Our models and those produced by Forzieri et al. (Forzieri et al. 2017) present plausible scenarios on the potential impact of climate change on human mortality and provide crucial information to public health officials, national governments, and international organizations. The time frames associated with climate change allow ample time for this 
potential health crisis to be averted.

Finally, we would like to point out that future research should not only transform excess mortality into life expectancy decrements. Given the influence of climate change in diseases and causes of death, it is imperative to quantify the extent to which climate change will derive in increasing costs for health care systems in these countries. The health care structures are being taxed by population aging (Rechel et al. 2009) and rising health care costs, yet it remains unclear how climate change will exacerbate these pressures.

\section{References}

Abel, G. J., Barakat, B., Samir, K. \& Lutz, W. (2016), 'Meeting the sustainable development goals leads to lower world population growth', Proceedings of the National Academy of Sciences 113(50), 14294-14299.

Arias, E., Heron, M. \& Tejada-Vera, B. (2013), 'United states life tables eliminating certain causes of death, 1999-2001.', National vital statistics reports: from the Centers for Disease Control and Prevention, National Center for Health Statistics, National Vital Statistics System 61(9), 1-128.

Barnett, J. \& Adger, W. N. (2007), 'Climate change, human security and violent conflict', Political geography 26(6), 639-655.

Beltrán-Sánchez, H., Preston, S. H. \& Canudas-Romo, V. (2008), 'An integrated approach to cause-of-death analysis: cause-deleted life tables and decompositions of life expectancy', Demographic research 19, 1323.

Cloyd, E., Moser, S. C., Maibach, E., Maldonado, J. \& Chen, T. (2016), 'Engagement in the third us national climate assessment: commitment, capacity, and communication for impact', Climatic change 135(1), 39-54.

Ebi, K. L., Kovats, R. S. \& Menne, B. (2006), 'An approach for assessing human health vulnerability and public health interventions to adapt to climate change', Environmental health perspectives 114(12), 1930. 
Forzieri, G., Cescatti, A., e Silva, F. B. \& Feyen, L. (2017), 'Increasing risk over time of weather-related hazards to the european population: a data-driven prognostic study', The Lancet Planetary Health 1(5), e200-e208.

Gerland, P., Raftery, A. E., Ševčíková, H., Li, N., Gu, D., Spoorenberg, T., Alkema, L., Fosdick, B. K., Chunn, J., Lalic, N. et al. (2014), 'World population stabilization unlikely this century', Science 346(6206), 234-237.

Haines, A., Kovats, R. S., Campbell-Lendrum, D. \& Corvalán, C. (2006), 'Climate change and human health: impacts, vulnerability and public health', Public health 120(7), 585596.

Hsiang, S., Kopp, R., Jina, A., Rising, J., Delgado, M., Mohan, S., Rasmussen, D., MuirWood, R., Wilson, P., Oppenheimer, M. et al. (2017), 'Estimating economic damage from climate change in the united states', Science 356(6345), 1362-1369.

Jiang, L. (2014), 'Internal consistency of demographic assumptions in the shared socioeconomic pathways', Population and environment 35(3), 261-285.

Keatinge, W., Donaldson, G., Cordioli, E., Martinelli, M., Kunst, A., Mackenbach, J., Nayha, S. \& Vuori, I. (2000), 'Heat related mortality in warm and cold regions of europe: observational study', Bmj 321(7262), 670-673.

Kendon, E. J., Roberts, N. M., Fowler, H. J., Roberts, M. J., Chan, S. C. \& Senior, C. A. (2014), 'Heavier summer downpours with climate change revealed by weather forecast resolution model', Nature Climate Change 4(7), 570.

Kovats, R., Ebi, K., Menne, B., Campbell-Lendrum, D., Canziani, O., Githeko, A., Kuhn, K., Le Sueur, D., Martens, P., McMichael, A. et al. (2003), Methods of assessing human health vulnerability and public health adaptation to climate change, WHOHealth CanadaUNEPWMO.

Lee, J. Y. \& Kim, H. (2017), 'Comprehensive assessment of climate change risks', The Lancet Planetary Health 1(5), e166-e167. 
Marmot, M., Allen, J., Bell, R. \& Goldblatt, P. (2012), 'Building of the global movement for health equity: from santiago to rio and beyond', The Lancet 379(9811), 181-188.

McMichael, A. J., Woodruff, R. E. \& Hales, S. (2006), 'Climate change and human health: present and future risks', The Lancet 367(9513), 859-869.

Melillo, J. M., Richmond, T. \& Yohe, G. (2014), 'Climate change impacts in the united states', Third National Climate Assessment .

of Disease Study 2015, G. B. (2017), 'lobal burden of disease study 2015 (gbd 2015) results', pp. Seattle, United States: Institute for Health Metrics and Evaluation (IHME), 2016. Available from http://ghdx.healthdata.org/gbd-results-tool. For terms and conditions of use, please visit http://www.healthdata.org/about/terms-and-conditions.

O’Neill, B. C., Kriegler, E., Ebi, K. L., Kemp-Benedict, E., Riahi, K., Rothman, D. S., van Ruijven, B. J., van Vuuren, D. P., Birkmann, J., Kok, K. et al. (2017), 'The roads ahead: narratives for shared socioeconomic pathways describing world futures in the 21st century', Global Environmental Change 42, 169-180.

Organization, W. H. (2015), World health statistics 2015, World Health Organization.

Pachauri, R. K., Allen, M. R., Barros, V. R., Broome, J., Cramer, W., Christ, R., Church, J. A., Clarke, L., Dahe, Q., Dasgupta, P. et al. (2014), Climate change 2014: synthesis report. Contribution of Working Groups I, II and III to the fifth assessment report of the Intergovernmental Panel on Climate Change, IPCC.

Parrish, R. G. (2010), 'Peer reviewed: Measuring population health outcomes', Preventing chronic disease $\mathbf{7}(4)$.

Patz, J. A., Campbell-Lendrum, D., Holloway, T. \& Foley, J. A. (2005), 'Impact of regional climate change on human health', Nature 438(7066), 310.

Rechel, B., Doyle, Y., Grundy, E., McKee, M., Organization, W. H. et al. (2009), 'How can health systems respond to population ageing'. 
Rigaud, K. K., De Sherbinin, A. M., Jones, B., Bergmann, J., Clement, V., Ober, K., Schewe, J., Adamo, S. B., McCusker, B., Heuser, S. et al. (2018), 'Groundswell: Preparing for internal climate migration'.

Rosenzweig, C., Solecki, W., Hammer, S. A. \& Mehrotra, S. (2010), 'Cities lead the way in climate-change action', Nature 467(7318), 909.

Salomon, J. A., Wang, H., Freeman, M. K., Vos, T., Flaxman, A. D., Lopez, A. D. \& Murray, C. J. (2012), 'Healthy life expectancy for 187 countries, 1990-2010: a systematic analysis for the global burden disease study 2010', The Lancet 380(9859), 2144-2162.

Samir, K. \& Lutz, W. (2017), 'The human core of the shared socioeconomic pathways: Population scenarios by age, sex and level of education for all countries to 2100', Global Environmental Change 42, 181-192.

United Nations (2015), 'World Population Prospects: The 2017 revision. methodology of the United Nations population estimates and projections'.

Wang, H., Dwyer-Lindgren, L., Lofgren, K. T., Rajaratnam, J. K., Marcus, J. R., LevinRector, A., Levitz, C. E., Lopez, A. D. \& Murray, C. J. (2012), 'Age-specific and sexspecific mortality in 187 countries, 1970-2010: a systematic analysis for the global burden of disease study 2010', The Lancet 380(9859), 2071-2094.

Wilson, A., Reich, B. J., Nolte, C. G., Spero, T. L., Hubbell, B. \& Rappold, A. G. (2017), 'Climate change impacts on projections of excess mortality at 2030 using spatially varying ozone-temperature risk surfaces', Journal of Exposure Science and Environmental Epidemiology 27(1), 118-124.

Zanobetti, A., O’neill, M. S., Gronlund, C. J. \& Schwartz, J. D. (2012), 'Summer temperature variability and long-term survival among elderly people with chronic disease', Proceedings of the National Academy of Sciences 109(17), 6608-6613. 